\title{
Second-Order Radiation Boundary Condition for Water Wave Simulation with Large Angle Incidence
}

\author{
Tai-Wen Hsu'; Yuan-Jyh Lan²; Ting-Kuei Tsay ${ }^{3}$; and Kuei-Pin Lin ${ }^{4}$
}

\begin{abstract}
A finite-element method (FEM) is used to simulate water wave propagation with large angle incidence at exterior boundaries. In this paper, the radiation boundary condition is expanded to a second-order approximation and a quadratic shape function is used in the FEM wave model. Cases used for verifications include wave scattering around a vertical cylinder and wave propagation over a submerged circular shoal with concentric contours. Numerical calculations based on this second-order radiation boundary condition are found to be in good agreement with theoretical and experimental results available. The numerical predictions show that this model has made a very good improvement over the first-order radiation boundary conditions for oblique wave incidence in coastal engineering.
\end{abstract}

DOI: $10.1061 /($ ASCE)0733-9399(2003)129:12(1429)

CE Database subject headings: Finite element method; Water waves; Wave propagation; Boundary conditions; Shores.

\section{Introduction}

For coastal engineering problems such as a beach nourishment project or a countermeasure of beach erosion control, it is desirable to get the information of wave conditions in the region of interest. The wave data are normally collected offshore and it is necessary to transfer these wave data on wave heights and wave propagation directions to the nearshore region. In recent years, many numerical wave models have been developed to calculate the combined wave transformations, resulting from wave refraction, diffraction, reflection, and shoaling. These models usually encountered two problems: the treatment of irregular boundary grids and large oblique wave incidence at the exterior boundary. Finite difference method (FDM) was one of the popular numerical techniques. Not only can its orthogonal rectangular meshes hardly match the irregular boundaries, but also the use of rough boundary grid near a structure reduces the accuracy of the local wave field near the boundaries. Recent development of the finiteelement method (FEM) is advantageous over the FDM in handling the complicated boundaries. The grid treatment for the FEM is more flexible than that in the FDM.

Earlier FEM for water wave propagation with oblique incidence generally employed the standard radiation boundary condition, which assumes scattered waves leave the domain in a direc-

\footnotetext{
${ }^{1}$ Professor, Dept. of Hydraulic and Ocean Engineering, National Cheng-Kung Univ., Tainan 701, Taiwan (corresponding author). E-mail: twhsu@mail.ncku.edu.tw

${ }^{2}$ Research Assistant, Dept. of Hydraulic and Ocean Engineering, National Cheng-Kung Univ., Tainan 701, Taiwan.

${ }^{3}$ Professor, Dept. of Civil Engineering, National Taiwan Univ., Taipei 106, Taiwan.

${ }^{4}$ Master, Dept. of Hydraulic and Ocean Engineering, National ChengKung Univ., Tainan 701, Taiwan.

Note. Associate Editor: Henry K. Stolarski. Discussion open until May 1, 2004. Separate discussions must be submitted for individual papers. To extend the closing date by one month, a written request must be filed with the ASCE Managing Editor. The manuscript for this paper was submitted for review and possible publication on October 1, 2001; approved on May 14, 2003. This paper is part of the Journal of Engineering Mechanics, Vol. 129, No. 12, December 1, 2003. CASCE, ISSN 0733-9399/2003/12-1429-1438/\$18.00.
}

tion perpendicular to the open boundaries at a far distance. However, the open boundaries are chosen artificially for convenience of numerical computations and usually are not very far from scatters due to concerns of numerical efficiency. Furthermore, variation in depth and existence of coastal structures near the boundaries affects wave direction, most scattered waves do not always leave the open boundaries as assumed. Recently, several techniques have been proposed to handle this problem. Berkhoff (1972) solved the elliptic mild-slope equation using the FEM and boundary integral techniques. Chen and Mei (1974) developed a hybrid finite-element model (HFEM), upon separating the computational domain into two regions, in which the interior was covered by a finite element grid, while the exterior of constant depth was modeled by analytical forms. Adopting the idea of a hybrid method, Bettess and Zienkiewicz (1977) suggested a new HFEM model using an infinite-element method in the exterior region. Instead of the analytical expansions of scattered waves in the exterior field, Chen and Tsay (1990) proposed a local radiation boundary condition to describe the scattered waves at the open boundary in the interior region.

The differences resulting from using the standard and local radiation boundary conditions were studied by simulating waves scattering around a circular island. The numerical wave models mentioned above require special treatment for the open boundaries with analytical forms or other numerical techniques, together with artificial boundaries in the form of a circle or semicircle. Constant depth is usually required beyond the exterior boundary. In reality, the water depths outside the exterior boundary in computational domain are hardly constant. Furthermore, the propagation direction of scattered waves is not always normal to the artificial boundaries. To overcome this problem, Panchang (2000) developed a technique that was based on a onedimensional representation to better simulate the effects of the exterior bathymetry. The results of the one-dimensional model were then interfaced along the open boundary of a twodimensional finite-element harbor wave model.

On the other hand, a radiation boundary condition (RBC) is specified such that there is no change of the velocity potential when a wave train crosses the boundary. Booij (1981) developed a numerical model, based on the original parabolic equation of 
Radder (1979) to handle the approximate boundary with variable water depth and large wave incidence angles at the open boundaries. Dingemans (1983) made use of the first-order Padé approximation for the radiation boundary condition, thus extending the angle of wave incidence to $56.5^{\circ}$. With a second-order Padé approximation, Kirby (1986) improved the applicability of the model to a large angle around $70^{\circ}$. Later, Dalrymple and Kirby (1988) developed a wave propagation model valid for incidence wave angles up to $90^{\circ}$, based on the assumption that the bottom contours were straight and parallel in a direction normal to the assumed direction of propagation. Additionally, Dalrymple et al. (1989) developed a "wide-angle" wave model for irregular bathymetry by a spectral Fourier method separating the contribution from component waves. Hsu et al. (1998) developed a FEM by adopting a binomial approximate to third order for the radiation boundary condition. The linear shape function used in their model was not suitable for differential terms in the second- and the third-order radiation boundary condition. Despite the shortcoming to improve its applicability for large wave incidence, Hsu's FEM model was helpful in treatment for open boundary.

In addition to coastal engineering, there are a large volume of existing papers dealing with the subject of $\mathrm{RBC}$ in computational physics, fluid dynamics, and applied mathematics. Givoli (1991) reviewed corresponding researches on the use of nonreflecting boundary conditions in wave problems. He also discussed boundary conditions, which are nonlocal in space, in time or both, and addressed some near future research directions in RBC problems. Givoli and Keller (1994) developed special two-dimensional finite elements for use near a boundary or an interface $\partial A$. The construction of the elements guarantees high-order $\left(C^{N}\right)$ regularity along $\partial A$ and $C^{0}$ regularity elsewhere. Their improvement shows that all the elements in the hierarchy are proved to be fully conforming. Tsynokov (1998) proposed an approach which can obtain highly accurate RBC in the form of certain nonlocal boundary operator equations. The new boundary conditions are geometrically universal, numerically inexpensive, and easy to implement along with the existing solvers. Hagstrom (1999) addressed a theory of exact boundary conditions for constant coefficient time-dependent problems. The calculation shows that the constant coefficient equations of wave theory on unbounded domains with sufficiently simple tails can be accurately solved at the same cost as solving a standard problem on the unbounded subdomain of interest. These achievements in different fields provide many new numerical ideas and techniques to treat the RBC problems. However, the conventional techniques for the RBC problems are still applied in the existing water wave models.

In this study, a finite-element wave model without the precondition of a special open boundary grid is presented. The radiation boundary condition is expanded to a second-order approximation using a quadratic shape function. This reduces unnecessary reflective waves numerically in the computational domain induced by inappropriate boundary condition. With this improvement, the proposed FEM offers satisfactory result of wave height distribution, subject to very large wave incidence at the open boundary. Numerical results for wave scattering around a vertical cylinder, and waves propagation over a submerged circular shoal are studied for verifications. The accuracy of present model is tested against available experimental, analytical and numerical results for waves with different large incident angles.

\section{Mathematical Formulation}

General geometry and regions of computation in this study are shown in Fig. 1, including the open boundaries (Curves AGF and

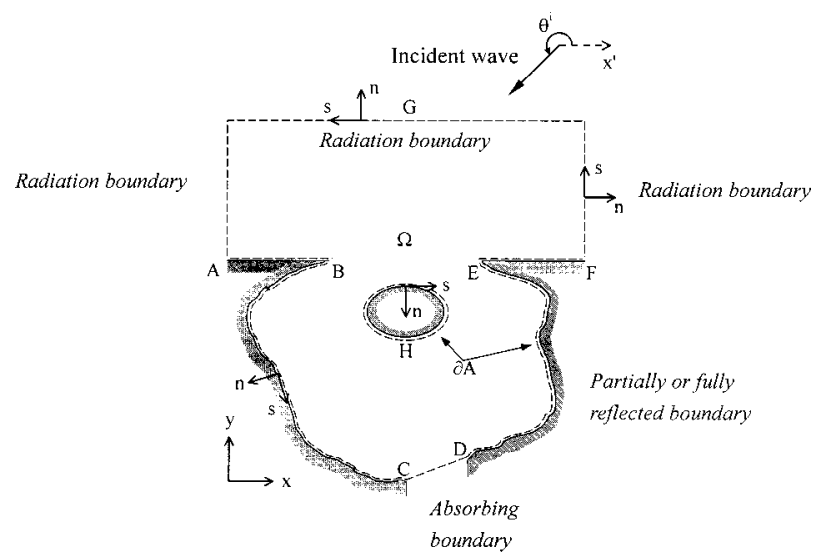

Fig. 1. General geometry and regions of computation

CD), shoreline (Curves ABC, DEF), and structure boundaries (Curves $\mathrm{H}$ ). The computational domain $\Omega$ is surrounded by dashes. Cartesian coordinate system $(x, y)$ is used, and the local coordinate system $(n, s)$ is adopted on boundaries in which $n$ and $s$, respectively, denote the normal and tangential direction. Counterclockwise rule is obeyed on the outer boundary of the domain to be solved and opposite rule observed on the internal boundary (Curve $\mathrm{H}$ ).

Considering wave propagation over slowly varying water depths, Berkhoff (1972) derived a two-dimensional mild-slope equation in the form

$$
\nabla \cdot\left(C C_{g} \nabla \eta\right)+C C_{g} k^{2} \eta=0
$$

where $\nabla=(\partial / \partial x, \partial / \partial y)=$ horizontal gradient operator; $\eta$ $=$ free-surface displacement in a complex form; $C=$ wave celerity; $C_{g}=$ group velocity; and $k=$ wave number. Eq. (1) is of elliptic type. Wave transformations such as wave shoaling, refraction, diffraction, and reflection can be obtained by solving Eq. (1) with suitable boundary conditions.

There are four types of boundaries in the simulation of wave transformation: full reflection, partial reflection, absorbing boundary, and radiation boundary. The radiation boundary condition is similar to the full absorption boundary condition, which allows specific wave trains transmitted through the boundary without any reflection. The former condition is referred to scattered waves while the latter is applied to total waves. A partial absorption boundary condition modified by radiation boundary condition is introduced by Behrendt (1985), giving

$$
\alpha \frac{\partial \eta}{\partial t}+C \frac{\partial \eta}{\partial r}=0, \quad \text { on } \partial A
$$

where $r=n \cos \theta+s \sin \theta=$ course of wave propagation, in which $\theta$ is the approaching wave angle at the boundary (see Fig. 2); $\alpha$

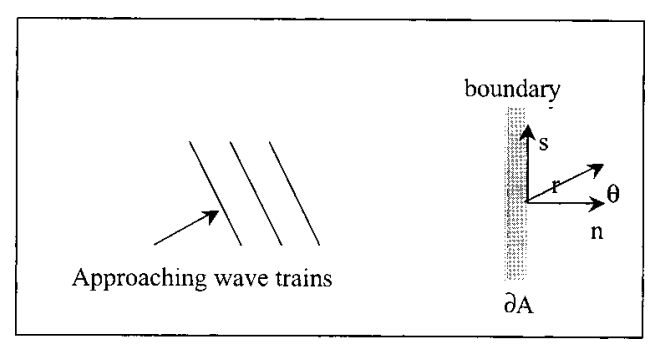

Fig. 2. Sketch of waves passing through boundary 


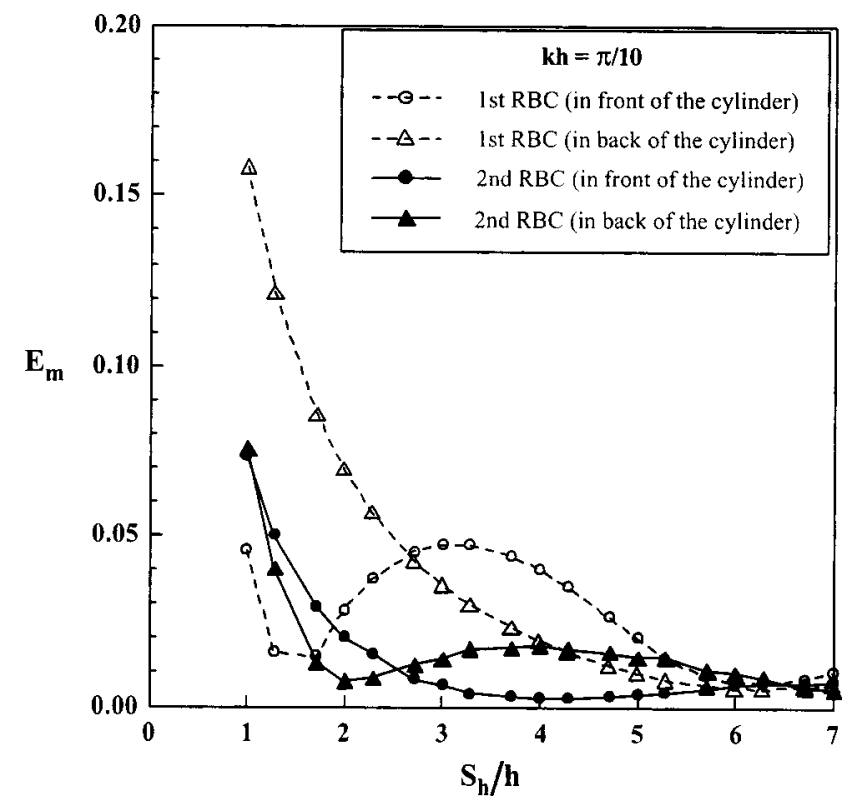

Fig. 3. Comparisons of averaged relative error $\left(E_{m}\right)$ of wave distribution versus relative axial distance $\left(S_{h} / h\right)$ for normal incidence $(k h=\pi / 10)$.

$=$ absorption coefficient defined as $(1-R) /(1+R)$; and $R$ $=$ reflection coefficient. When $\alpha=0$ and $\alpha=1$, the above boundary condition represents full reflection and full passing through boundary conditions, respectively. If $0<\alpha<1$, Eq. (2) becomes the partial-reflection boundary condition.

Any free-surface displacement of chronometric wave component $\eta$, with unit amplitude on the boundary can be assumed as

$$
\eta=e^{i\left(k_{n} n+k_{s} s-\omega t\right)}
$$

in which $\omega(=2 \pi / T)=$ wave radian frequency; $T=$ wave period; and $k_{n}=k \cos \theta$ and $k_{s}=k \sin \theta=$ local wave number components

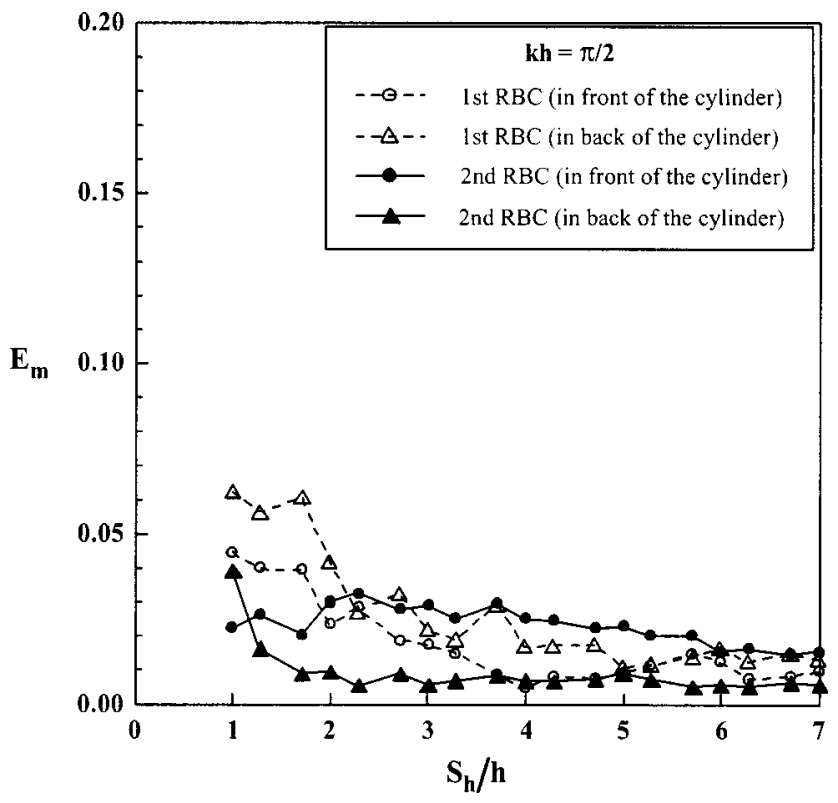

Fig. 4. Comparisons of averaged relative error $\left(E_{m}\right)$ of wave distribution versus relative axial distance $\left(S_{h} / h\right)$ for normal incidence $(k h=\pi / 2)$.

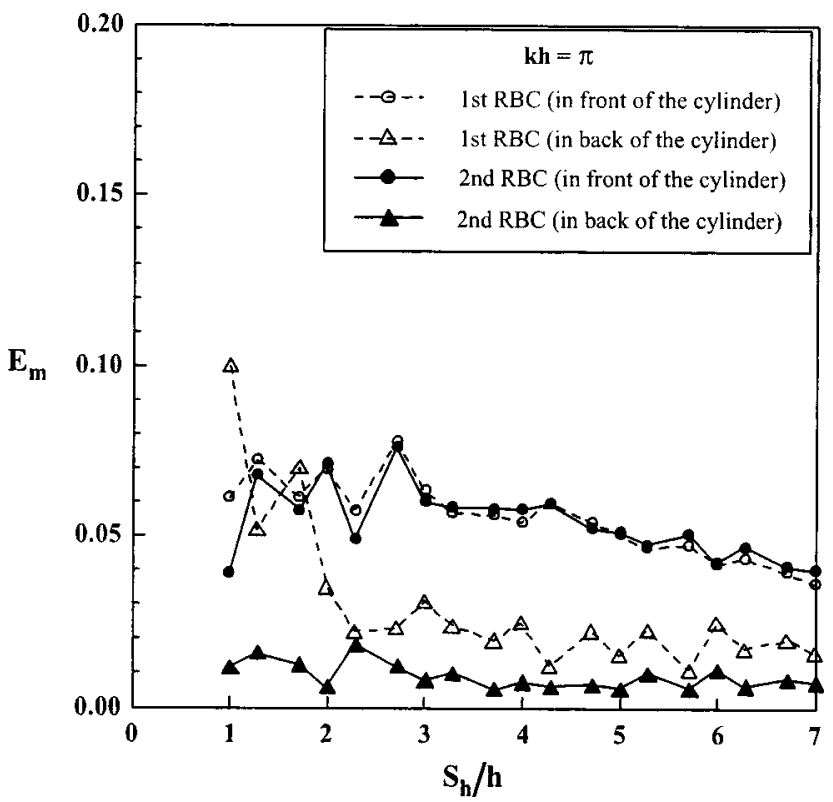

Fig. 5. Comparisons of averaged relative error $\left(E_{m}\right)$ of wave distribution versus relative axial distance $\left(S_{h} / h\right)$ for normal incidence $(k h=\pi)$.

in the $n$ and $s$ direction, respectively. Substituting Eq. (3) into Eq. (2), the partial reflection boundary condition on $\partial A_{n^{+}}$for total wave components becomes

$$
\frac{\partial \eta}{\partial n}-i \alpha k_{n} \eta=0, \quad \text { on } \partial A_{n^{+}}
$$

where

$$
k_{n}=k \cos \theta=k \sqrt{1-\sin ^{2} \theta}=k \sqrt{1-\left(\frac{k_{s}}{k}\right)^{2}}
$$

Using a binomial expansion method, the right-hand-side term in Eq. (5) can be expanded as

$$
k \sqrt{1-\left(\frac{k_{s}}{k}\right)^{2}}=k\left[1-\frac{1}{2}\left(\frac{k_{s}}{k}\right)^{2}+\mathrm{O}\left(\frac{k_{s}}{k}\right)^{4}\right]
$$

If the approaching wave trains pass through the exterior boundary in the $n$ direction almost perpendicular to the boundary $\partial A$, i.e., $\sin \theta=k_{s} / k=0$, Eq. (6) becomes almost unity and Eq. (4) is reduced to

$$
\frac{\partial \eta}{\partial n}-i \alpha k \eta=0, \quad \text { on } \partial A_{n^{+}}
$$

which is the partial reflection boundary condition to the first order. Note that, in the traditional wave models of Berkhoff (1972), Chen and Mei (1974), Bettess and Zienkiewicz (1977), and Tsay and Liu (1983), the radiation boundary condition is adopted by Eq. (7) with $\alpha=1$ for the scattered waves.

In this study, Eq. (6) is approximated up to the first two terms. Substituting the relationship of $\partial^{2} \eta / \partial s^{2}=-k_{s}^{2} \eta$ from Eq. (3), the partial reflection boundary condition for total waves to a secondorder can be expressed as

$$
\frac{\partial \eta}{\partial n}-i \alpha k\left(\eta+\frac{1}{2 k^{2}} \frac{\partial^{2} \eta}{\partial s^{2}}\right)=0, \quad \text { on } \partial A_{n^{+}}
$$

when the radiation boundary condition is used at the driving boundaries where incident wave $\eta^{i}$ is specified. Free-surface dis- 


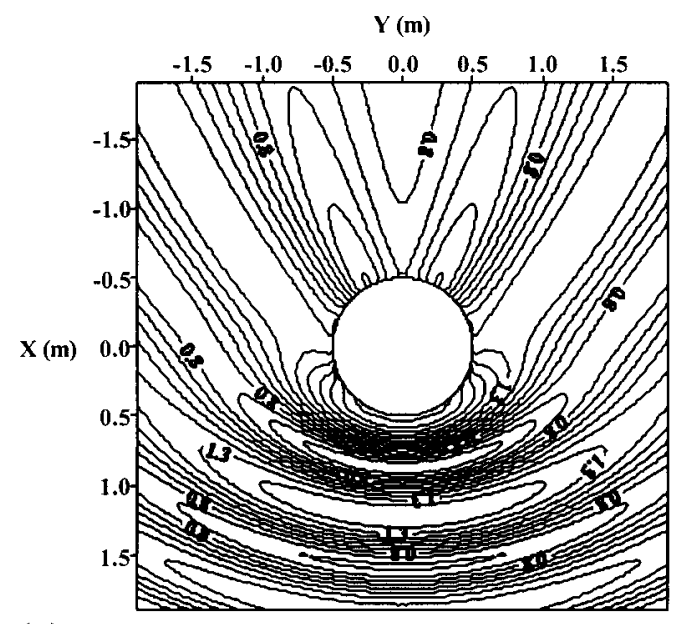

(a)

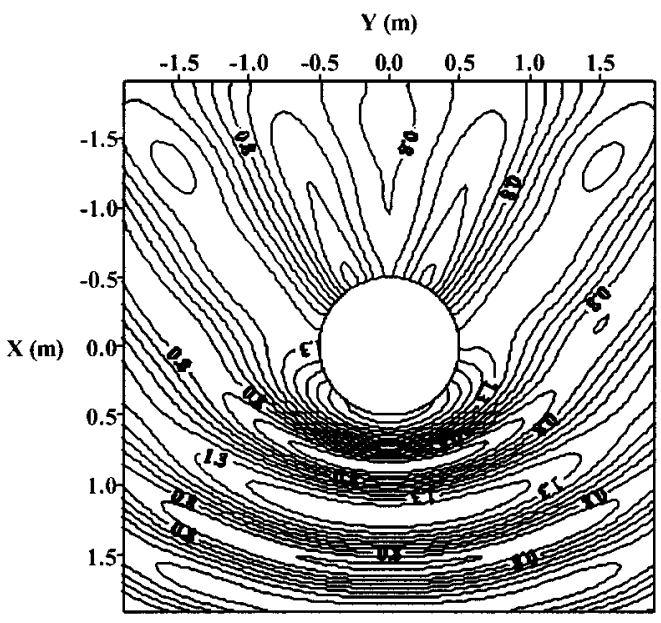

(b)

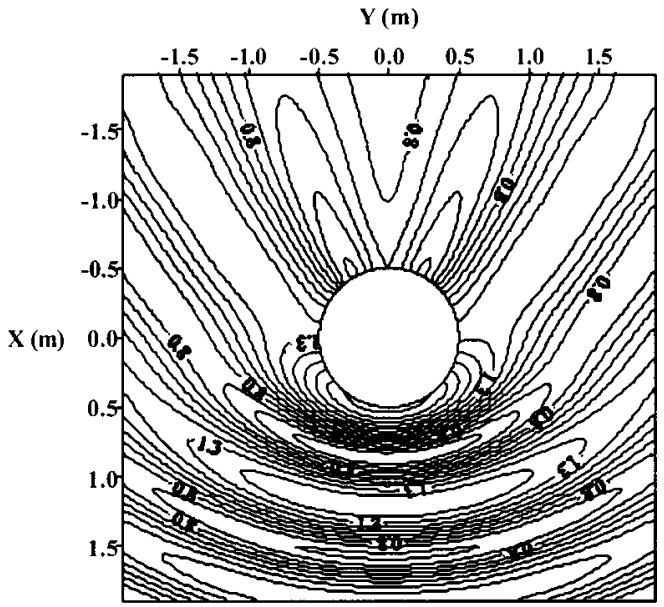

(c)

Fig. 6. Wave height patterns around vertical cylinder for normal incidence

placement of total waves $\eta$ on given boundaries is equal to $\eta^{i}$ $+\eta^{s}$. The outgoing scattered waves $\eta^{s}$ should propagate across this boundary and remain unaffected, which means $\eta^{s}$ also satisfies the radiation boundary condition in a second-order form

$$
\frac{\partial \eta^{s}}{\partial n}-i k\left(\eta^{s}+\frac{1}{2 k^{2}} \frac{\partial^{2} \eta^{s}}{\partial s^{2}}\right)=0
$$

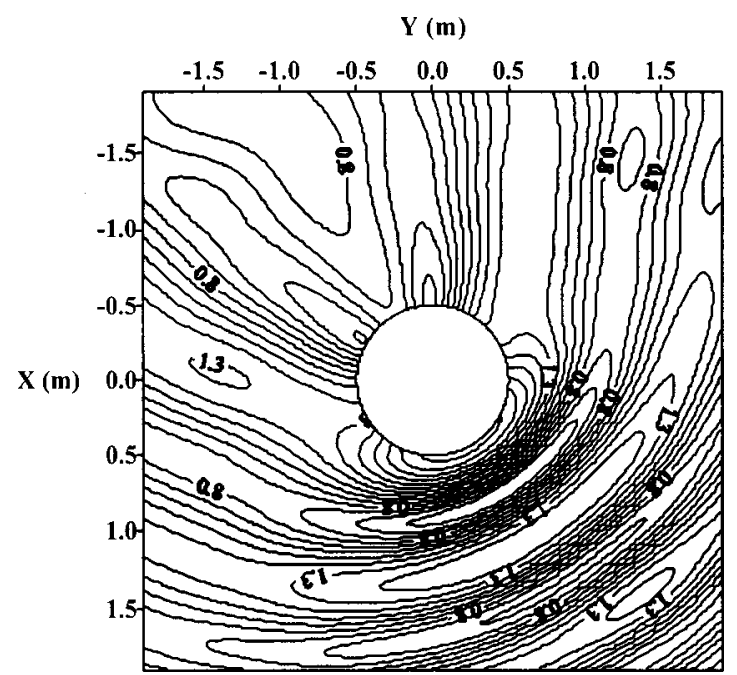

(a)

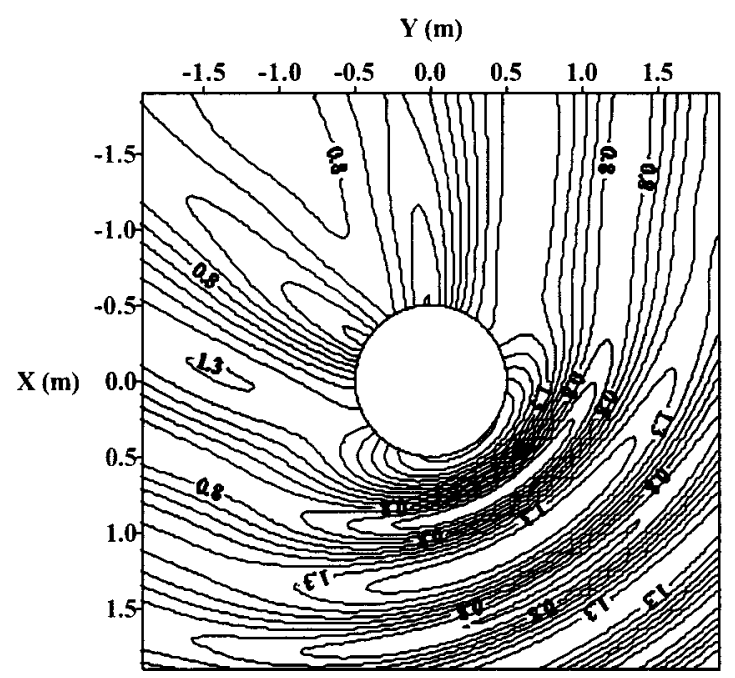

(b)

Fig. 7. Wave height patterns around vertical cylinder for wave incidence at $30^{\circ}$

The traditional radiation boundary condition can be easily obtained by approximating Eq. (9) to the first order. Thus the absorbing boundary conditions for scattered waves to the first and second order, respectively, can be written as

$$
\begin{gathered}
\frac{\partial \eta}{\partial n}=\left(\frac{\partial \eta^{i}}{\partial n}-i k \eta^{i}\right)+i k \eta \\
\frac{\partial \eta}{\partial n}=\left(\frac{\partial \eta^{i}}{\partial n}-i k \eta^{i}-\frac{i}{2 k} \frac{\partial^{2} \eta^{i}}{\partial s^{2}}\right)+\left(i k \eta+\frac{i}{2 k} \frac{\partial^{2} \eta}{\partial s^{2}}\right)
\end{gathered}
$$

A general type of radiation boundary conditions to the first and second order for any arbitrarily chosen open boundaries is introduced in this study and can be expressed, respectively, as

$$
\begin{gathered}
\frac{\partial \eta}{\partial n}=\left(\frac{\partial \eta^{i}}{\partial n}-i \alpha k \eta^{i}\right)+i \alpha k \eta \\
\frac{\partial \eta}{\partial n}=\left(\frac{\partial \eta^{i}}{\partial n}-i \alpha k \eta^{i}-\frac{i \alpha}{2 k} \frac{\partial^{2} \eta^{i}}{\partial s^{2}}\right)+\left(i \alpha k \eta+\frac{i \alpha}{2 k} \frac{\partial^{2} \eta}{\partial s^{2}}\right)
\end{gathered}
$$

Should $\eta^{i}$ become zero, i.e., $\eta=\eta^{s}$, boundary conditions (12) and (13) will be reduced to the partial absorption boundary con- 


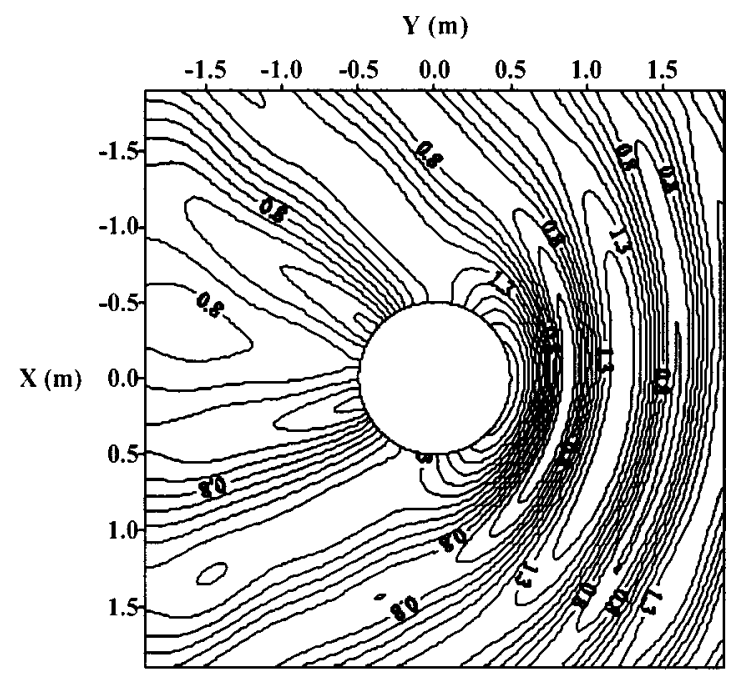

(a)

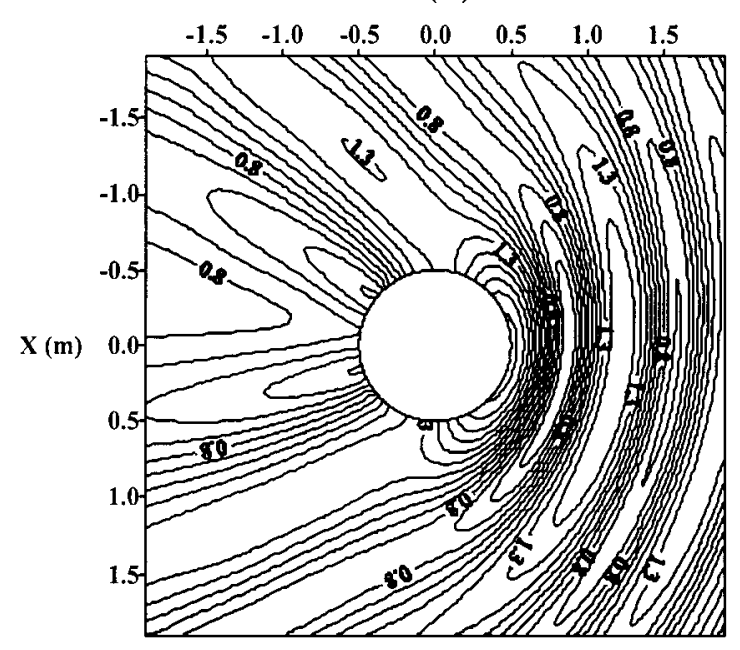

(b)

Fig. 8. Wave height patterns around vertical cylinder for wave incidence at $80^{\circ}$

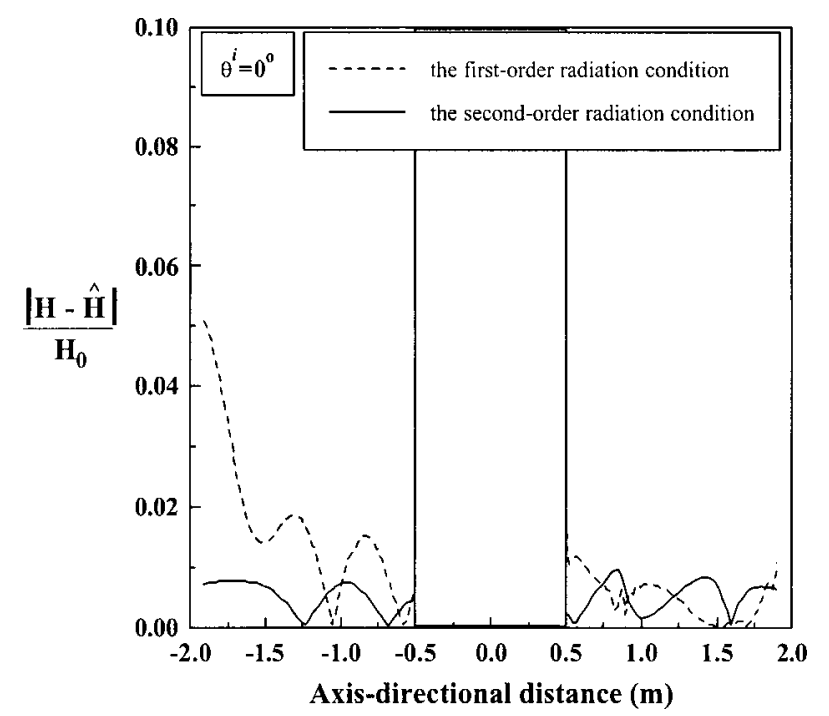

Fig. 9. Comparisons of relative errors of wave distribution in axial direction across vertical cylinder for normal incidence

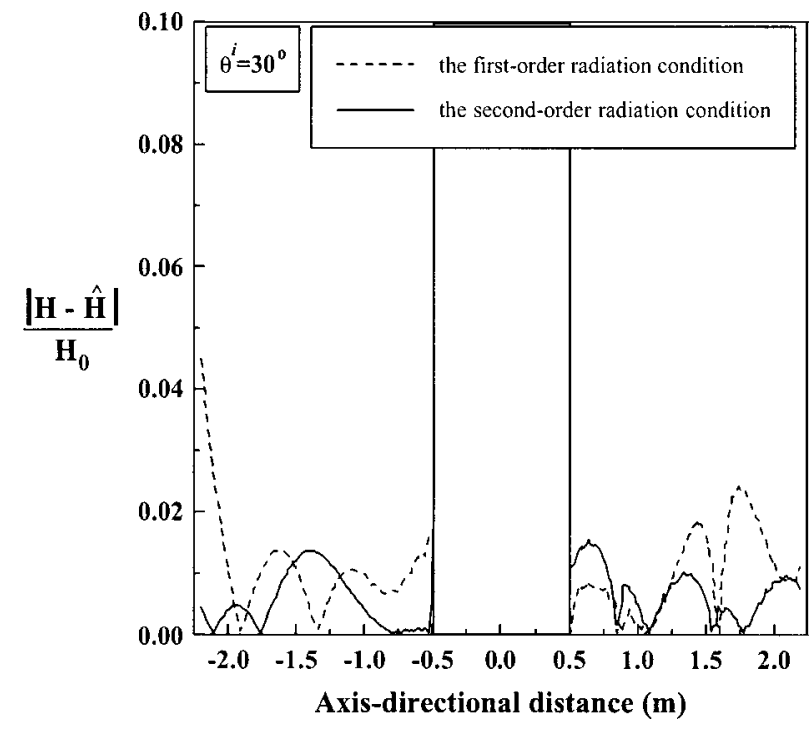

Fig. 10. Comparisons of relative errors of wave distribution in axial direction across vertical cylinder for $30^{\circ}$ angle of incidence

dition given by Eqs. (7) and (8), respectively. The boundary conditions (12) and (13) can also be reduced to the radiation boundary conditions (10) and (11) by setting $\alpha=1$.

It is noted that the radiation boundary conditions of Eqs. (8), (11), and (13) can be reduced to the special case of the general form as

$$
\frac{\partial \eta}{\partial n}+\sum_{j=1}^{N+1} \frac{\partial^{j}}{\partial n^{j}}\left(a_{j}(n) \frac{\partial^{j} \eta}{\partial n^{j}}\right)=0, \quad \text { on } \partial A
$$

where $N(N=0$ in this study) represents the minimum degree of higher-order regularity $\left(C^{N}\right)$ on the open boundary (Givoli and Keller 1994). We notice that our treatment possesses $C^{0}$ continuity everywhere when evaluating the corresponding integrals. In principle, one is able to apply a higher-order RBC along $\partial A$ by using the appropriate $C^{N}$ element. In fact, the present work is

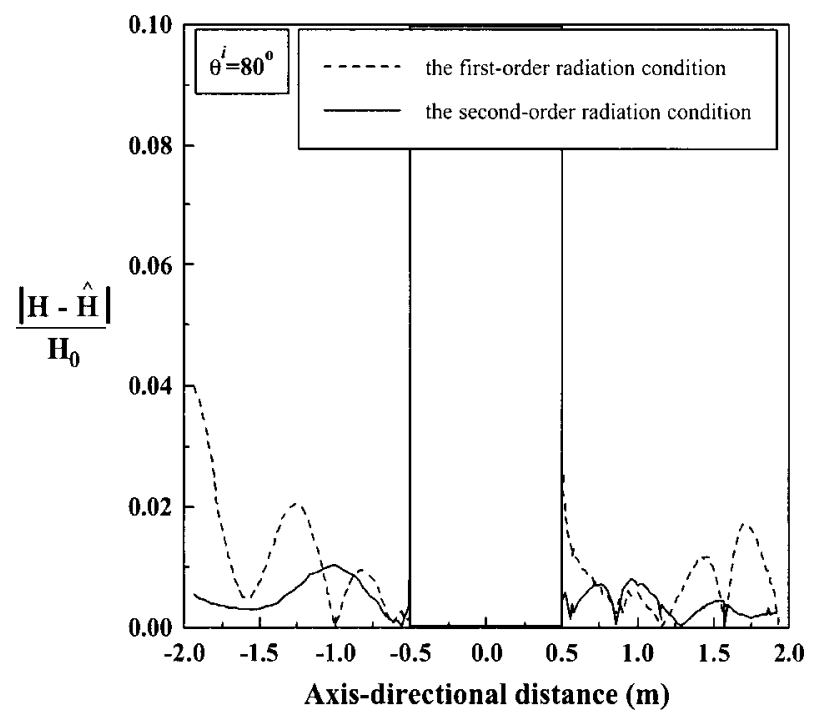

Fig. 11. Comparisons of relative errors of wave distribution in axial direction across vertical cylinder for $80^{\circ}$ angle of incidence 


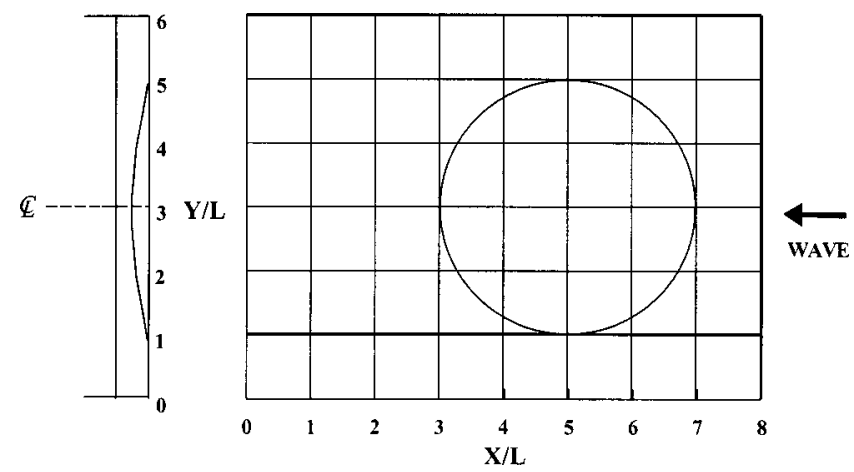

Fig. 12. Arrangement of circular shoal (Ito and Tanimoto 1972)

strongly related to coastal engineering. The proposed scheme does differ from available schemes in the element formulations associated with RBC problems.

\section{Finite-Element Model}

The boundary value problem described above is solved using a finite-element method. Using a weighted residual method and the divergence theorem, the elliptic type mild-slope equation of Eq.

(1) can be written into the weighted residual equation as

$$
\iint_{\Omega}\left[C C_{g} \nabla \eta \cdot \nabla \phi_{i}-k^{2} C C_{g} \eta \phi_{i}\right] d A=\int_{\partial A} C C_{g} \frac{\partial \eta}{\partial n} \phi_{i} d s
$$

where $\phi_{i}=$ weighting function. In this study, Galerkin finiteelement method is employed. Eq. (15) contains the domain integral in $\Omega$ and the boundary integral on $\partial A$. Because of the second-order partial differential terms in Eqs. (8), (11), and (13), a quadratic shape function is required to describe the free-surface displacement $\eta$ in each element. Six-point triangular elements are used to discretize the computational domain. In the numerical formulation, the physical variables $q\left(q=\eta\right.$, and $C C_{g}$, etc. $)$ is expressed as

$$
q=\sum_{j=1}^{6} \phi_{j} q_{j}
$$

Substituting Eqs. (16)-(15), after some algebraic manipulations, we finally obtain a system of simultaneous dispersed weighted residual equations for free-surface displacement

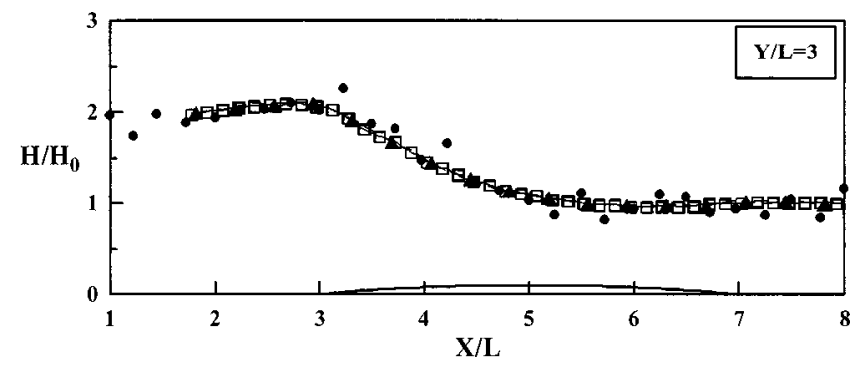

Fig. 13. Comparison for calculated wave heights and experimental results at $Y / L=3(\boldsymbol{O}$ : Experimental data; $-\boldsymbol{\Delta}-$ : Numerical solution for first-order radiation boundary condition; and $-\square-$ : Numerical solution for second-order radiation boundary condition)

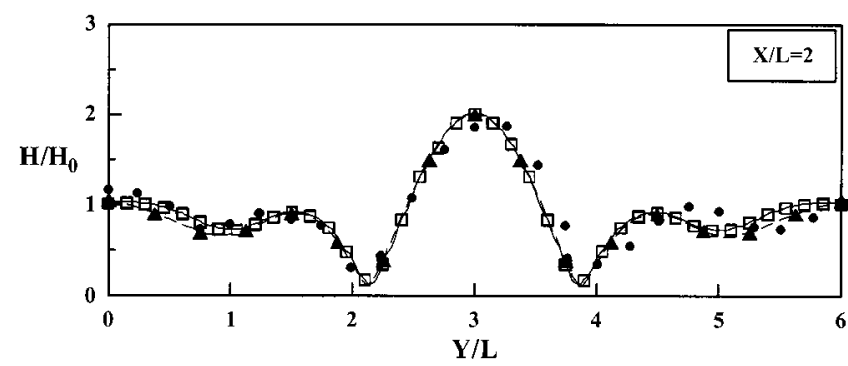

Fig. 14. Comparison for calculated wave heights and experimental results at $X / L=2(\bullet$ : Experimental data; $-\boldsymbol{\Delta}-$ : Numerical solution for first-order radiation boundary condition; and $-\square-$ : Numerical solution for second-order radiation boundary condition)

$$
\sum_{j=1}^{N} K_{i j} \eta_{j}=F_{i}, \quad i=1 \sim N
$$

where $F_{i}=F_{1}+F_{2}+F_{3}$, and

$$
\begin{aligned}
& K_{i j}=\sum_{m=1}^{N}\left(C C_{g}\right)_{m} \iint_{\Omega} \nabla \phi_{j} \cdot \nabla \phi_{i} \phi_{m} d A \\
& -\sum_{m=1}^{N}\left(\omega^{2} C_{g} / C\right)_{m} \iint_{\Omega} \phi_{i} \phi_{j} \phi_{m} d A \\
& -\sum_{m=1}^{N}(i \alpha)\left(k C C_{g}\right)_{m} \int_{\partial A} \phi_{i} \phi_{j} \phi_{m} d s \\
& -\sum_{m=1}^{N}\left(\frac{i \alpha}{2}\right)\left(C C_{g} / k\right)_{m} \int_{\partial A} \phi_{i} \phi_{j, s s} \phi_{m} d s \\
& F_{1}=\int_{\partial A} C C_{g} \frac{\partial \eta^{i}}{\partial n} \phi_{i} d s \\
& =\sum_{j=1}^{N}\left[\sum_{m=1}^{N}(i)\left(k C C_{g}\right)_{m} \int_{\partial A} \phi_{i} \phi_{j} \phi_{m} d s\right] \\
& \times \cos \left(\theta^{i}-\theta^{\prime}\right){ }_{j} \eta_{j}^{i} \\
& F_{2}=\int_{\partial A}(-i \alpha) k C C_{g} \eta^{i} \phi_{i} d s \\
& =\sum_{j=1}^{N}\left[\sum_{m=1}^{N}(-i \alpha)\left(k C C_{g}\right)_{m} \int_{\partial A} \phi_{i} \phi_{j} \phi_{m} d s\right] \eta_{j}^{i}
\end{aligned}
$$

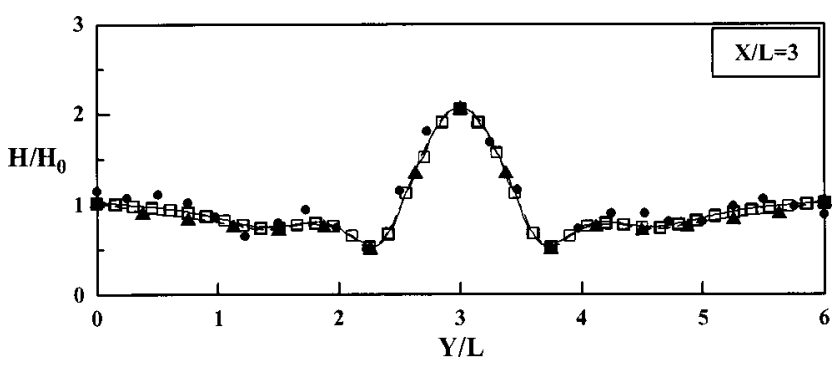

Fig. 15. Comparison for calculated wave heights and experimental results at $X / L=3$ (०: Experimental data; $-\mathbf{\Delta}-$ : Numerical solution for first-order radiation boundary condition; and $-\square-$ : Numerical solution for second-order radiation boundary condition) 
$\mathbf{Y} / \mathbf{L}$

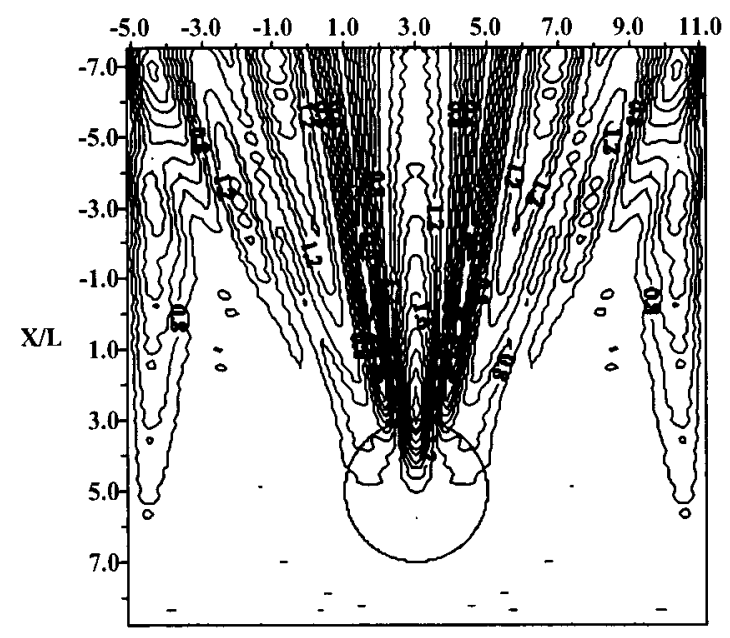

(a)

$\mathbf{Y} / \mathbf{L}$

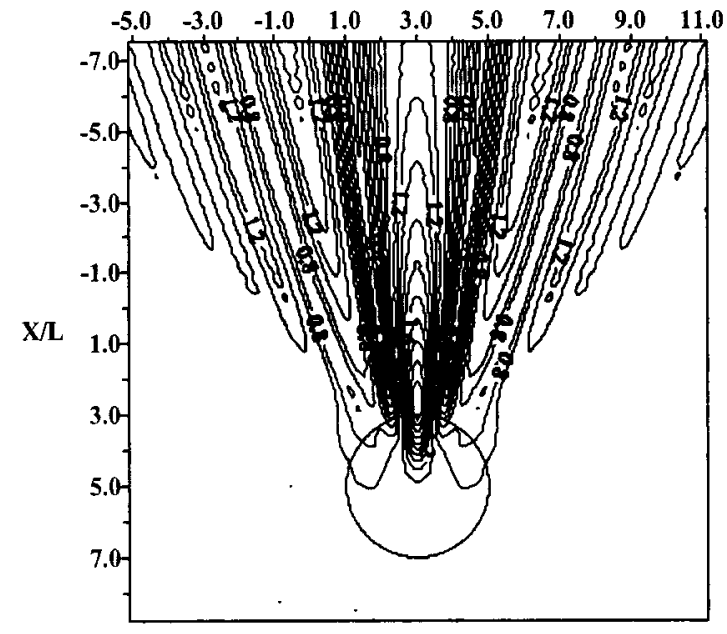

(b)

Fig. 16. Wave height patterns behind submerged circular shoal for normal incidence

$$
\begin{aligned}
F_{3}= & \int_{\partial A}-\frac{i \alpha}{2 k} C C_{g} \frac{\partial^{2} \eta^{i}}{\partial s^{2}} \phi_{i} d s \\
= & \sum_{j=1}^{N}\left[\sum_{m=1}^{N}\left(\frac{i \alpha}{2}\right)\left(k C C_{g}\right)_{m} \int_{\partial A} \phi_{i} \phi_{j} \phi_{m} d s\right] \\
& \times\left[\sin \left(\theta^{i}-\theta^{\prime}\right)\right]^{2} \eta_{j}^{i}
\end{aligned}
$$

in which $\theta^{i}=$ incident wave angle; $\theta^{\prime}=$ angle between $n$ axis of local coordinate and $x$ axis of the fixed Cartesian coordinate. The matrices resulting from the last term of Eq. (18) apparently destroy the symmetry of the finite-element matrix equations. However, the problem of asymmetric matrices in numerical scheme can be approximately ignored by the recent computational capacity.

Each of the integrals in Eqs. (18)-(21) can be calculated as the sum of contributions furnished by each element in numerical meshes. The numerical meshes in this study are constituted by triangular elements $\Omega^{e}(x, y)$ with the quadratic function $\phi_{i}^{e}$. For convenience, an invertible transformation between an arbitrary
$\mathrm{Y} / \mathrm{L}$

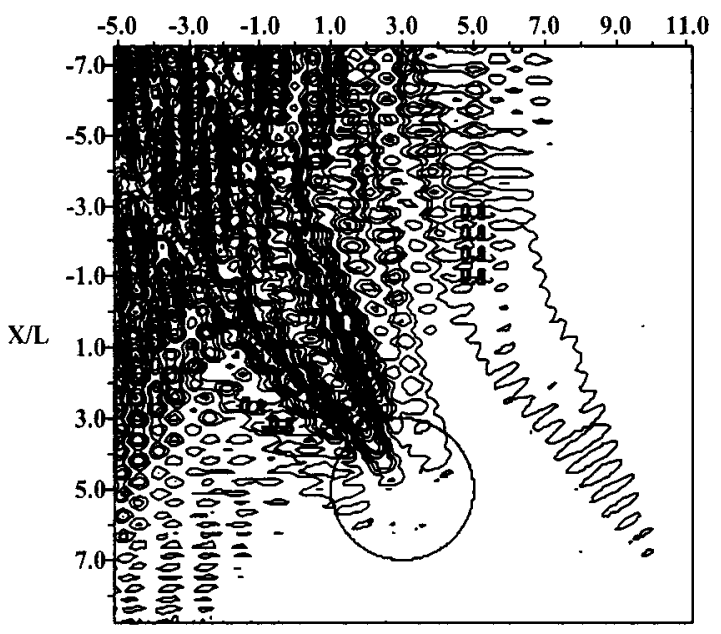

(a)

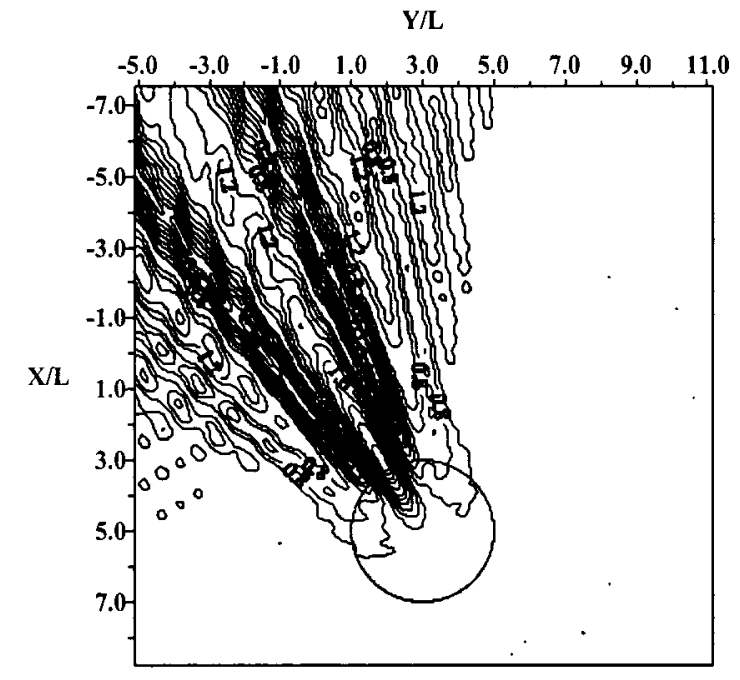

(b)

Fig. 17. Wave height patterns behind submerged circular shoal for $30^{\circ}$ of wave incidence

triangular element $\Omega^{e}(x, y)$ and a master element $\hat{\Omega}(\xi, \eta)$ of simple shape is introduced in the model. The development of shape functions for a six-point quadratic triangle in natural coordinates referred to a master element is a standard FEM technology which can be found in the Becker et al. (1981) literature.

\section{Results and Discussion}

To verify the applicability and validity of the new approach, the present finite-element wave model is applied to two test cases. The first is a simulation of wave scattering around a vertical cylinder surrounded by a sea of constant depth. The second one is for waves propagating over a submerged circular shoal. A fixed computational domain is used with variable wave incidences for numerical computations. Since water depths outside the shoal or surrounding the cylinder are constant, changes of wave incidences with respect to the computational domain must not affect wave field. Comparisons using the first-order and second-order radiation boundary conditions are then discussed in details. All tests are conducted for nonbreaking waves. 
$\mathrm{Y} / \mathrm{L}$

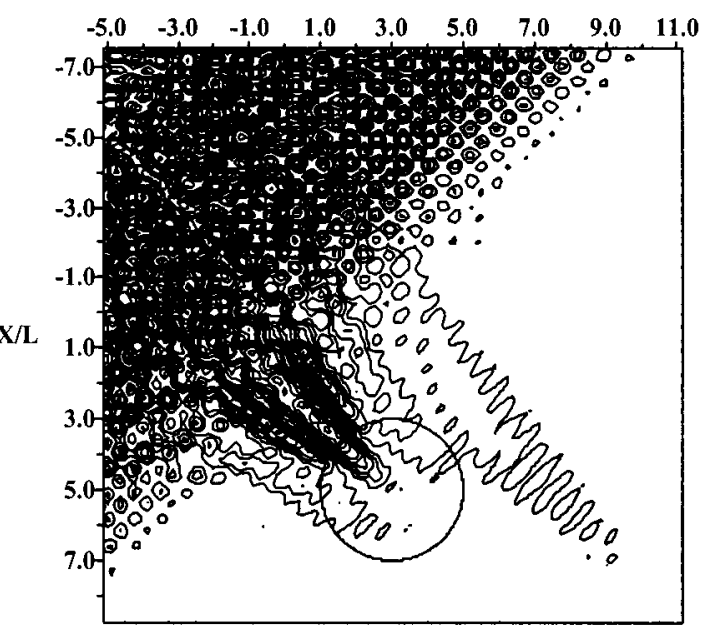

(a)

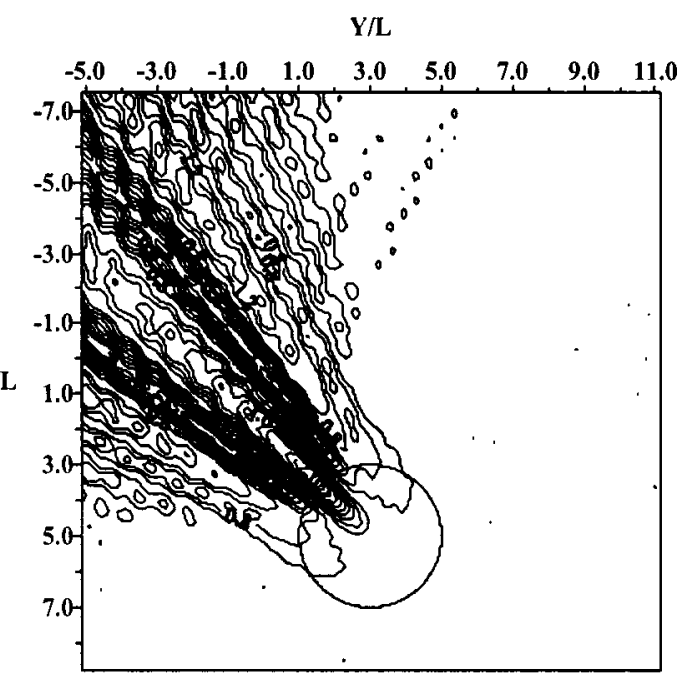

(b)

Fig. 18. Wave height patterns behind submerged circular shoal for $45^{\circ}$ of wave incidence

\section{Wave Scattering around a Vertical Cylinder}

A vertical cylinder set in constant depth that causes radial scattering is simulated to make a comparison of different approximations of the radiation boundary condition. There was an analytical solution to the problem of waves diffracted around a vertical cylinder in water of constant depth (MacCamy and Fuchs 1954). Analytical solutions are symmetrical to wave propagating direction regardless of computational domains. The solution is used as the first test case, where the incident wave height $H_{0}$ is $0.053 \mathrm{~m}$, diameter of cylinder $D$ is $1.0 \mathrm{~m}$, and water depth $h$ is $0.35 \mathrm{~m}$. A fixed square computational domain rather than a conventionally circular one is chosen for severe tests by changing wave incident angles. The first-order and second-order radiation boundary conditions, respectively, are implemented in the FEM wave model. The radiation boundary condition is applied to boundary where waves are incoming and the absorbing boundary conditions are applied to boundaries where waves are outgoing. The circumference of the cylinder is set to the full reflection boundary condition.
$\mathrm{Y} / \mathrm{L}$

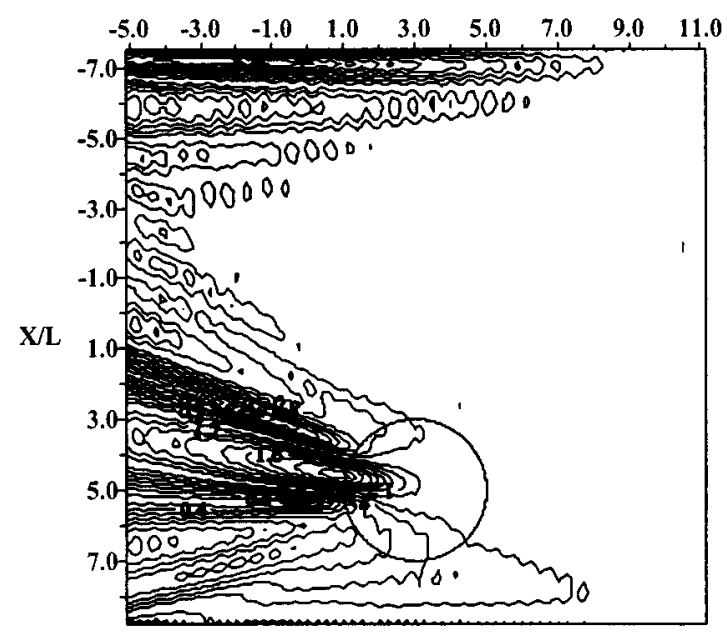

(a)

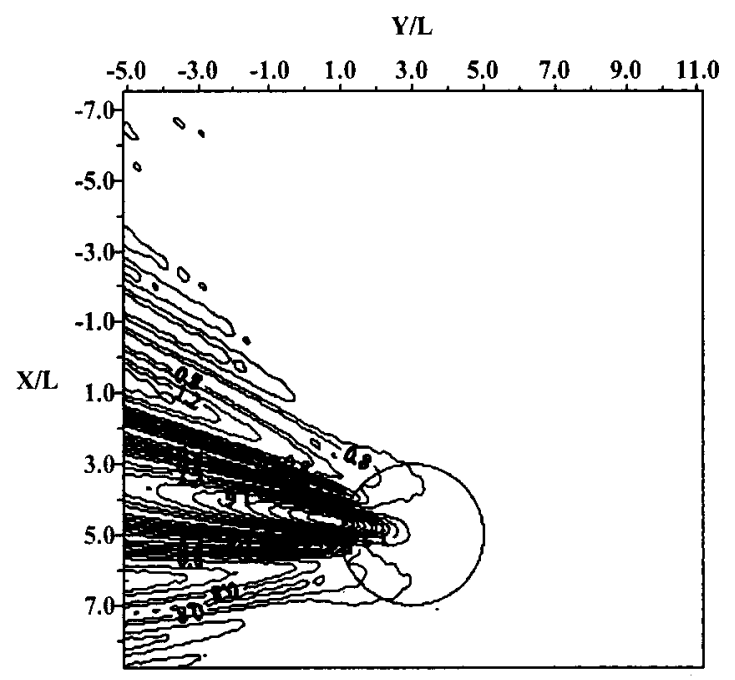

(b)

Fig. 19. Wave height patterns behind submerged circular shoal for $80^{\circ}$ of wave incidence

The range of the computational domain is tested first in this study. The distance in the $x$ direction between the vertical cylinder and the artificial boundary is defined as $S_{h}$. The wave height pattern will approach to a constant value as the distance $S_{h}$ reaches a sufficiently large value. An averaged relative error $E_{m}$ is used to determine the range of the computational domain and defined as

$$
E_{m}=\frac{1}{N} \sum_{i=1}^{N} \frac{\left|H_{i}-\hat{H}_{i}\right|}{\hat{H}_{i}}
$$

where $\hat{H}=$ wave height calculated from analytic solution; and $H$ $=$ value calculated from the numerical model. Three kinds of wave conditions, $k h=\pi / 10$ (shallow water wave), $\pi / 2$ (intermediate water wave), and $\pi$ (deep water wave), are chosen to calculate the averaged relative error $E_{m}$ versus the relative axial distance $S_{h} / h$. The calculated results of the three cases are showed in Figs. 3, 4, and 5, respectively. For $S_{h} / h \geqslant 4$, the values of $E_{m}$ are small than $6 \%$ for all calculated wave cases. A reasonable minimum off-scatter distance $S_{h} / h=4$ is thus used in the following computational cases. 
Three obliquely incident wave angles, $0^{\circ}, 30^{\circ}$, and $80^{\circ}$, are used as the input wave conditions in the study, where the wave period $T$ is $1.0 \mathrm{~s}(k h=0.49 \pi)$. It should be noted that the case of $90^{\circ}$ wave incidence is equivalent to the case of $0^{\circ}$ wave incidence as far as fixed square computational domain is concerned. The computed wave height patterns are shown in Figs. 6, 7, and 8. Fig. 6 gives the computed wave height patterns around a circular cylinder for incident waves in negative $x$-axis direction and perpendicular to the lower bottom open boundary. The results show that the wave height pattern based on the second-order radiation boundary condition [Fig. 6(c)] becomes identical to that of the analytical solution [Fig. 6(a)]. The results given by the firstorder boundary condition are different apparently near the artificial boundaries. Due to the axisymmetry of the vertical cylinder in constant water depths, predictions of the wave-scattering pattern around the cylinder should be independent of the incident wave angle. Figs. 7 and 8 for the wave pattern computed from incident angle of $30^{\circ}$ and $80^{\circ}$ with respect to the $x$-axis, respectively. The results using the second-order radiation boundary condition appear symmetric to propagating direction of incident waves [Figs. 7(b) and 8(b)]. However, the patterns for the firstorder radiation boundary condition, as in Figs. 7(a) and 8(a) are asymmetric near the lower bottom open boundaries. From Fig. $8(\mathrm{a})$, the first-order radiation boundary condition seems to produce a more reasonable wave pattern than the case of $30^{\circ}$. This is because that wave incidence of $80^{\circ}$ from the $x$-axis is equivalent to wave incidence of $-10^{\circ}$ from the $y$-axis. The numerical inconsistency revealed in Figs. 7(a), and 8(a) is due to the firstorder radiation boundary condition, which assumes the scattering waves perpendicularly propagate across open boundaries. The second-order radiation boundary condition is thus demonstrated to allow waves to obliquely propagate through open boundaries.

Comparisons of the relative error of calculated wave height in the axial direction across the cylinder and analytic results for different incident angles are presented in Figs. 9, 10, and 11, respectively, where $|H-\hat{H}| / H_{0}$ is the error of relative wave height. It is shown that numerical results based on first-order radiation boundary condition are quite different from the theory. With a fixed computational domain, the larger incident wave angle the bigger the difference between the theory and that of the first-order approximation to open boundary conditions. For the second-order radiation boundary condition, the calculated wave heights are in good agreement with the analytical solutions.

\section{Waves Propagating over a Submerged Circular Shoal}

Waves propagating over a submerged circular shoal resting on a flat bottom are simulated using the first-order and the secondorder radiation boundary conditions, respectively. This experiment was first carried out by Ito and Tanimoto (1972). Geometry of the shoal is given in Fig. 12. The water depth $h$ is expressed as

$$
\begin{array}{cc}
h=h_{m}+\frac{h_{0}-h_{m}}{R^{2}} r^{2} & \text { for } r<R \\
h=h_{0} & \text { for } r \geqslant R
\end{array}
$$

where $r=\sqrt{\left(x-x_{m}\right)^{2}+\left(y-y_{m}\right)^{2}} ; \quad\left(x_{m} / L, y_{m} / L\right)=(5,3)=$ center of the circular shoal; $h_{0}=0.15 \mathrm{~m}=$ constant depth outside the shoal; $h_{m}=0.05 \mathrm{~m}=$ water depth at the crest of the shoal; and $R$ $=0.8 \mathrm{~m}=$ radius of the shoal. The incident wave height, wave period, and wavelength are $H_{0}=0.0208 \mathrm{~m}$, period $T=0.511 \mathrm{~s}$, and wavelength $L=0.4 \mathrm{~m}$, respectively. For numerical computations to verify present second-order radiation boundary condition, incident wave angles ranging from $0^{\circ}$ to $80^{\circ}$ are used. A fixed rectangular computational domain, in which the $x$ - and $y$-coordinates are in the range of $-7.5 \leqslant x / L \leqslant 8.75$ and $-5.125 \leqslant y / L \leqslant 11.125$, respectively, is chosen for severe tests by changing wave incident angles. The absorbing boundary conditions are used on the lower bottom open boundary, and the radiation boundary conditions are use on the upward and lateral ones. Solutions of wave field must be also symmetrical to wave propagation direction.

Comparisons between the calculated wave heights and the experimental results for normal incidence are presented in Figs. 13, 14 , and 15 , respectively. It is shown that both numerical results using first- and second-order radiation boundary conditions, respectively, agree very well with the experiment results. Furthermore, wave height patterns in a fixed computational rectangle for $\theta^{i}=0^{\circ}, 30^{\circ}, 45^{\circ}$, and $80^{\circ}$ calculated by first- and second-order radiation boundary conditions are shown in Figs. 16, 17, 18, and 19 , respectively. Owing to the axisymmertic nature of the submerged circular shoal, predictions of the wave-focusing pattern behind the shoal should be independent of the incident wave angle. Although both the first- and second-order radiation boundary conditions reproduce the axisymmetric wave height contours for $0^{\circ}$ wave incidence, as in Figs. 16(a and b), respectively, the first-order radiation boundary condition displays unnecessary wave reflection near the lateral open boundaries, while the second-order one does not. Similar trend of lateral reflections for $\theta^{i}=30^{\circ}, \theta^{i}=45^{\circ}$, and $\theta^{i}=80^{\circ}$, respectively resulting from the first-order radiation boundary condition approximations are, respectively, shown in Figs. 17(a), 18(a), and 19(a). It is worth noting that wave patterns due to the first-order radiation boundary condition are distorted, even though it predicts a favorable focusing wave pattern. The distortion of wave height patterns increases with increasing incident wave angle for the first-order radiation boundary condition approximation. From Figs. 16 to 19, it indicates that the wave patterns based on the second-order radiation boundary condition really allow wave transmitting so as to reduce wave reflection from the lateral and artificial boundaries back into the limited computational domain.

\section{Conclusions}

A FEM model is proposed for water wave propagation under combined refraction, diffraction, and reflection incorporating large wave incidence. The governing mild-slope equation is of elliptic type, subject to a second-order radiation boundary condition for arbitrarily chosen open boundaries to improve performance of strong wave obliquity in numerical calculations. A quadratic shape function is used to represent the physical variable in model development. In this paper, the present results using the first- and second-order radiation boundary conditions are verified in two typical tests: wave propagation around a vertical cylinder and wave passing over a submerged circular shoal. It is found that the second-order approximation for the radiation boundary condition improves the FEM performance, as well as extends ranges of incident angles of waves at arbitrarily chosen open boundaries.

\section{Acknowledgment}

This study was financially supported by National Science Council, Taiwan, under Grant No. NSC-89-2611-E-006-071.

\section{References}

Becker, E. B., Carey, G. F., and Oden, J. T. (1981). Finite elements: An introduction, Prentice-Hall, Englewood Cliffs, N.J. 
Behrendt, L. (1985). "A finite element model for water wave diffraction including boundary absorption and bottom friction." Series Paper 37, Institute of Hydrodynamics and Hydraulic Engineering, Univ. of Denmark, Lyngby, Denmark.

Berkhoff, J. C. W. (1972). "Computation of combined refractiondiffraction." Proc., 13th Int. Conf. on Coastal Engineering, ASCE, New York, 471-490.

Bettess, P., and Zienkiewicz, O. C. (1977). "Diffraction and refraction of surface waves using finite and infinite element." Int. J. Numer. Methods Eng., 11(8), 1271-1290.

Booij, N. (1981). "Gravity waves on water with non-uniform depth and current." Rep. No. 81-1, Dept. of Civil Engineering, Delft Univ. of Technology, The Netherlands.

Chen, B. S., and Tsay, T. K. (1990). "Application of local radiation condition to water-wave numerical modeling." Proc., 12th Conf. on Ocean Engineering in ROC, Taichung, Taiwan, 1-9 (in Chinese).

Chen, H. S., and Mei, C. C. (1974). "Oscillation and wave force on an offshore harbor." Rep. No. 190, Ralph M. Parsons Laboratory, Massachusetts Institute of Technology, Cambridge, Mass.

Dalrymple, R. A., and Kirby, J. T. (1988). "Models for very wide-angle water waves and wave diffraction." J. Fluid Mech., 192, 33-50.

Dalrymple, R. A., Suh, K. D., Kirby, J. T., and Chag, J. W. (1989). "Models for very wide-angle water waves and wave diffraction. Part 2: irregular bathymetry." J. Fluid Mech., 201, 299-322.

Dingemans, M. W. (1983). "Verification of numerical wave propagation method with field measurements: CREDIZ verification Haringvliet." Rep W488, Pt. 1, Delft Hydraulic Laboratory, Delft, The Netherlands.

Givoli, D. (1991). "Non-reflecting boundary conditions." J. Comput. Phys., 94(1), 1-29.
Givoli, D., and Keller, J. B. (1994). "Special finite elements for use with high-order boundary conditions." Comput. Methods Appl. Mech. Eng., 119(3-4), 199-213.

Hagstrom, T. (1999). "Radiation boundary conditions for the numerical simulation of waves." Acta Numerica, 8, 47-106.

Hsu, T. W., Tsay, T. K., Yan, C. C., and Chen, B. S. (1998). "Simulations on wave field in nearshore zone by finite element method." Proc., 20th Ocean Engineering Conf. in ROC, Keelung, Taiwan, 491-499 (in Chinese).

Ito, Y., and Tanimoto, K. (1972). "A method of numerical analysis of wave propagation application to wave diffraction and refraction." Proc., 13th Int. Conf. on Coastal Engineering, ASCE, New York, 503-522.

Kirby, J. T. (1986). "Rational approximations in the parabolic equation method for water waves." Coastal Eng., 10(6), 503-522.

MacCamy, R. C., and Fuchs, R. A. (1954). "Wave force on piles: a diffraction theory." Institute of Engineering Research, Waves Investigation Laboratory, Series 3, Issue 334, Berkeley, Calif.

Panchang, V., Chen, W., Xu, B., Schlenker, K., Demirbilek, Z., and Okihiro, M. (2000). "Exterior bathymetric effects in elliptic harbor wave models." J. Waterw., Port, Coastal, Ocean Eng., 126(2), 71-78.

Radder, A. C. (1979). "On the parabolic equation method for water wave propagation." J. Fluid Mech., 95, 159-176.

Tsay, T. K., and Liu, P. L.-F. (1983). "A finite element model for wave refraction and diffraction." Appl. Ocean. Res., 5(1), 30-37.

Tsynkov, S. V. (1998). "Numerical solution of problems on unbounded domains." Appl. Numer. Math., 27(4), 465-532. 